\title{
The reinforcement of polystyrene and poly(methyl methacrylate) interfaces using alternating copolymers
}

\author{
M.J. Arlen, M.D. Dadmun* \\ Department of Chemistry and Neutron Sciences Consortium, The University of Tennessee, Knoxville, TN 37996-1600, USA
}

Received 3 June 2003; received in revised form 8 August 2003; accepted 20 August 2003

\begin{abstract}
Asymmetric double cantilever beam studies are presented that document the ability of alternating copolymers to strengthen a polymer/polymer interface. For polystyrene/poly(methyl methacrylate) interfaces, these results show that the alternating copolymer is the least effective sequence distribution of a linear copolymer at strengthening the polystyrene/poly(methyl methacrylate) interface, where the copolymers that are compared all have similar molecular weight and composition. The results also demonstrate that the effect of copolymer molecular weight on the ability of the copolymer to strengthen an interface is controlled by the balance between the increased entanglements and decreased miscibility of the copolymer with the homopolymers with increasing molecular weight.
\end{abstract}

(C) 2003 Elsevier Ltd. All rights reserved.

Keywords: Polymer blends; Compatibilizers; Copolymer

\section{Introduction}

Ideally, two different polymers that exhibit different physical properties may be blended at different volume fractions to yield new materials with tunable properties. If the system forms a miscible mixture, an intimate combination or entanglement of (at least) two polymer chains exists in the blend [2]. The final properties of the material then depends on the synergistic behavior of the two different polymer chains at different volume fractions. The physical properties that may be improved include impact, chemical and thermal resistance, modulus, elongation at break, and adhesion [3]. However, the problem with most polymer pairs is that they are immiscible. Most polymer pairs that are mixed during processing yield phase separated systems that possess coarse phase morphology, where the interface between phases is sharp. The resulting phase separated polymer blends yield poor mechanical properties that are usually inferior to either of the homopolymers, due to the lack of entanglements between phases.

In order to create a useful material, it is often required that these phase-separated blends be compatibilized to increase the interfacial adhesion at the biphasic interface,

\footnotetext{
* Corresponding author. Tel.: + 1-8659746582; fax: + 1-8659743454.

E-mail address: dad@utk.edu (M.D. Dadmun).
}

improve dispersion, and increase their overall properties. Several methods for compatibilizing polymer mixtures such as addition of copolymer, crosslinking agents, or the introduction of specific interactions (i.e. hydrogen bonding) have been studied [1-5]. For an immiscible polymer blend, it has been shown that a copolymer that is made from the same monomers as the blend components will improve the mechanical properties of the blend [6-10]. For instance, work by Kramer, [11-20] Russell, [21,22] Riess, [23-27] Teyssié, [28-32] and others [33-38] have examined the ability of linear copolymers to strengthen a biphasic interface, including copolymers with various sequence distributions, including tapered, diblock and random copolymers.

Of particular interest to this work is results by Kramer and coworkers for polystyrene/poly(2-vinyl pyridine) (PS/P2VP) interfaces reinforced with diblock and tri-block copolymers containing $\mathrm{S}$ and $2 \mathrm{VP}$, where the measured interfacial fracture toughness $\left(G_{\mathrm{c}}\right)$ was observed to increase with the number of blocks in the copolymer that form entanglements with the homopolymers [12]. In a separate study, PS/P(2VP) interfaces were also reinforced by multiblock copolymers composed of $\mathrm{S}$ and poly(4-hydroxystyrene). This system also demonstrated that the pentablock copolymer was a more effective interfacial modifier than the 
triblock copolymer, [11] in agreement with our recent results $[4-6,34]$ that suggest that blocky copolymers are the most effective interfacial strengtheners.

The effectiveness of random copolymers to reinforce polymers interfaces has also been widely investigated [11, $12,15,21,22,33-38]$ and was found to be dependent on the monomer composition [22]. In the case of $\mathrm{P}\left(\mathrm{S}_{f}\right.$-ran$\left.\mathrm{MMA}_{1-f}\right)$, where $f$ is the monomer composition, the compositionally asymmetric $\mathrm{P}\left(\mathrm{S}_{0.7}\right.$-ran-MMA $)$ displayed the greatest reinforcement of the PS/PMMA interface out of a range of $\mathrm{P}(\mathrm{S}-$ ran-MMA) compositions studied [21].

As with all compatibilizers, the random copolymer is believed to strengthen the interface by weaving back and forth between polymer phases. However, the exact number of interfacial crossings a random copolymer possesses is difficult to experimentally determine. Therefore, the ability of a random copolymer to reinforce an interface has been correlated to the resulting interfacial width, $w_{\mathrm{i}}$, of the polymer/polymer interfaces in the presence of a copolymeric compatibilizer to better understand the underlying reinforcement mechanism. Russell and coworkers used neutron reflectivity (NR) to correlate asymmetric double cantilever beam (ADCB) results of PS/PMMA interfaces reinforced with $\mathrm{P}\left(\mathrm{S}_{f}\right.$-ran-MMA $\left.\mathrm{MM}_{1-f}\right)$ to its interfacial strengthening [21]. Results demonstrate that the strongest modifier, $\mathrm{P}\left(\mathrm{S}_{0.7}\right.$-ran-MMA), provides an interfacial profile that is symmetric.

ADCB studies by Kramer on PS/P2VP interfaces reinforced with $\mathrm{P}(\mathrm{S}$-ran-2VP) agree with these results. In these experiments, the addition of a compositionally symmetric $\mathrm{P}(\mathrm{S}-\mathrm{ran}-2 \mathrm{VP})$ resulted in the greatest increase in $G_{\mathrm{c}}$ of the interface and forward elastic recoil spectroscopy showed that this copolymer provided a symmetric interface [15]. In a separate study, investigation of the fractured ADCB beams using forward recoil spectroscopy determined that in a system with multiple interfaces fracture always occurs at the interface with the smallest $w_{\mathrm{i}}$ [16].

Recent work in our laboratory also demonstrates that the efficiency of a copolymeric compatibilizer depends intimately on its sequence distribution [4-6,34]. Both theory and experimental results demonstrate that the copolymer that forms more entanglements with the two homopolymers will be the most effective interfacial modifier, particularly in terms of strengthening the interface $[4-10,33,34]$. Conceptually, increasing the number of times the copolymer crosses the interface increases the strength of biphasic interfaces, if each crossing results in an entanglement. However, the correlation between sequence distribution and interfacial crossings is not clear, and thus, we are interested in understanding the complete correlation of a copolymers sequence distribution to its ability to strengthen a biphasic interface between two homopolymers. In this manuscript, the range of linear copolymer sequence distribution examined as compatibilizers will be extended to include alternating copolymers. The results document the ability of $\mathrm{P}(\mathrm{S}$-alt-MMA) to reinforce PS/PMMA interfaces when placed at the interface and the importance of the copolymer molecular weight on this process. The $G_{\mathrm{c}}$ of PS/PMMA interfaces modified with five $\mathrm{P}(\mathrm{S}$-alt-MMA) copolymers with molecular weight varying from $8.50 \times 10^{4}$ to $2.55 \times 10^{6} \mathrm{~g} / \mathrm{mol}$ were measured as a function of copolymer layer thickness using ADCB. These results are then compared to ADCB results for other $\mathrm{P}(\mathrm{S}-$-co-MMA) used to reinforce the PS/PMMA interface. To more thoroughly understand the molecular weight effects of the interfacial modification process by alternating copolymers, the miscibility of the $\mathrm{P}(\mathrm{S}$-alt-MMA) copolymer in both d-PMMA and PS was also investigated. NR measurements were also performed on compatibilized styrene/methyl methacrylate interfaces that are similar to the ADCB samples. This information provides a more detailed understanding of the interfacial structure of the reinforced interfaces and is correlated to the reinforcement processes of the $\mathrm{P}(\mathrm{S}$-altMMA) copolymers.

\section{Experimental}

Asymmetric double cantilever beam tests. The ADCB test was used to quantify the increased strength of the polystyrene/poly(methyl methacrylate) interface with the addition of the alternating copolymers. This technique provides a quantitative measure of the fracture toughness, $G_{\mathrm{c}}$, of polymer/polymer interfaces that are compatibilized by the addition of the copolymer. The procedure entails driving a razor blade through the interface between homopolymer layers and measuring the length of the resulting crack, which is inversely proportional to $G_{\mathrm{c}}$ [39-44]. Thus, this technique provides a method by which the ability of different copolymer sequence distributions and architectures to increase the fracture toughness between two polymers can be quantified and compared.

Sample preparation. The materials used in this project and their properties are listed in Table 1. All the polymers used in this project were purchased from Polymer Source, except for the deuterated polystyrene (d-PS). The 50/50 alternating copolymers are referred to by altXXX, where ' $\mathrm{XXX}$ ' denotes the weight average molecular weight of the copolymer divided by 1000 . All polymers were used as

Table 1

Molecular weight characteristics of polymers

\begin{tabular}{lrrr}
\hline Polymer & \multicolumn{1}{l}{$M_{\mathrm{n}}$} & \multicolumn{1}{l}{$M_{\mathrm{w}}$} & PDI \\
\hline PS & 140,000 & 230,000 & 1.64 \\
d-PS & 104,000 & 109,000 & 1.05 \\
PMMA & 62,500 & 100,000 & 1.60 \\
d-PMMA & 327,300 & 360,000 & 1.10 \\
Alt85 & 36,000 & 85,200 & 2.37 \\
Alt96 & 46,200 & 96,100 & 2.08 \\
Alt108 & 48,500 & 108,600 & 2.24 \\
Alt497 & 235,000 & 497,000 & 2.21 \\
Alt2m & $1,700,000$ & $2,550,000$ & 1.50 \\
\hline
\end{tabular}


received. The d-PS was synthesized in our lab using highvacuum anionic polymerization methods [45]. Purified benzene and $5 \mathrm{~g} \mathrm{~d}-8$ styrene were placed in an anionic reactor and $4.8 \times 10^{-5} \mathrm{~mol}$ of sec-butyl lithium was dissolved in hexane and introduced to the reactor. The molecular weight characteristics of this deuterated copolymer was determined by size exclusion chromatography using a Waters Gel Permeation Chromatograph equipped with ultrastyragel columns with a refractive index detector and tetrahydrofuran as the elution solvent, and are summarized in Table 1. Polystyrene homopolymer standards were used for molecular weight calibration.

The ADCB samples were prepared by annealing PS and PMMA at $150{ }^{\circ} \mathrm{C}$ in a vacuum oven for at least $48 \mathrm{~h}$ to remove any residual solvent or monomer. PS and PMMA homopolymers were compression molded and cut into strips that were $1 \times 6.5 \times 0.23$ and $1 \times 6.5 \times 0.25 \mathrm{~cm}$, respectively. The beam thickness ratio of 1.1 was used in this study as it was determined to be the optimal beam geometry by both Winey and Brown for similar ADCB studies on PS/PMMA beams reinforced with $\mathrm{P}(\mathrm{S}$-co-MMA) copolymers [46]. This geometry yields a slightly negative phase angle which deflects the crack towards the craze resistant PMMA, causing the propagating crack to grow at the biphasic interface, fracturing the joint with minimal crazing.

Tri-layer samples were constructed by placing alternating copolymers at the PS/PMMA interface for six copolymer layer thicknesses ranging from 35 to $300 \mathrm{~nm}$. The copolymer layers were spin cast from toluene solutions onto clean glass slides at a spinning rate of $2500 \mathrm{rpm}$, for $30 \mathrm{~s}$. The sides of the glass slides were scored and the films were delicately floated on distilled water. The water was drained and the films were carefully placed onto the PS strips. The bi-layer samples were then dried at $80{ }^{\circ} \mathrm{C}$ in a vacuum oven for at least $24 \mathrm{~h}$ to remove excess water or toluene. The thickness of the copolymer films was controlled by the concentration of the solutions that varied from $0.85-5.3 \mathrm{wt} \%$. The correlation between solution concentration and the copolymer film thickness was determined using a nulling ellipsometer [34]. Dry PMMA strips were then placed on top of the bi-layer to complete the tri-layer samples. The samples were then annealed for two hours at $150{ }^{\circ} \mathrm{C}$ under slight pressure $(<10 \mathrm{lb})$. The annealing time and temperature used in this studied were chosen to be consistent with similar studies of PS/PMMA interfaces reinforced with other $\mathrm{P}(\mathrm{S}-\mathrm{co}-\mathrm{MMA})$ structures for the purpose of comparing results $[34,36]$.

The $G_{\mathrm{c}}$ was measured by fracturing PS/PMMA beams with a razor that was inserted at the interface and driven into the interface at a speed of $0.20 \mathrm{~mm} / \mathrm{min}$. Pictures of the propagating crack were then captured every 1-2 min using a video camera and the pictures were imaged on a computer. Crack lengths were measured from the leading edge of the razor blade to the crack tip after the system had reached dynamic equilibrium. In this project, 4-6 tri-layer samples were constructed for each copolymer layer thickness, where
30-70 measurements of the crack length were used to determine $G_{\mathrm{c}}$ for each sample.

Specular neutron reflectivity. Trilayer samples for NR measurements were constructed on clean silicon wafers. The wafers were washed three times, using alternate washings of acetone and toluene to remove any organic material from the surface. The wafers were then placed in an ultra-violet ozone oven for $15 \mathrm{~min}$ to provide a uniform silicon oxide surface. The bottom layer was constructed by spin casting a $1.8 \mathrm{wt} \%$ d-PMMA toluene solution onto the silicon wafer to yield a film thickness of approximately $65 \mathrm{~nm}$. The middle layer, approximately $35 \mathrm{~nm}$ thick, was constructed by spin casting $1.0 \mathrm{wt} \%$ solutions of Alt497 and Alt $2 \mathrm{~m}$ in toluene onto separate clean glass slides. The films were then floated and placed onto separate d-PMMA films. To complete the tri-layer samples, a $1.8 \mathrm{wt} \%$ d-PS solution was spin cast onto clean glass slides to yield thin films approximately $65 \mathrm{~nm}$ thick, which were floated and placed on the bilayer samples. The tri-layer samples were then annealed in a vacuum oven for two hours at $150{ }^{\circ} \mathrm{C}$.

NR measurements for the trilayer samples were performed on the NG-7 reflectometer at the National Center for Neutron Research at the National Institute of Standards and Technology in Gaithersburg, MD. The neutron wavelength was $4.768 \AA$ with a wavelength spread $(\Delta \lambda / \lambda)$ of 0.2. Reflectivity measurements were scanned by transversing the incident neutron beam through the air/polymer interface. The scattering length density (SLD) profiles were calculated from model fits of the measured reflectivity data using the MIRROR program, where the interfaces are modeled as two 1/2 Gaussians [48].

Miscibility. The miscibility of the five different $M_{\mathrm{w}} \mathrm{P}(\mathrm{S}$ alt-MMA) in both d-PMMA and PS was examined to more thoroughly understand the strengthening behavior. To test the miscibility, 1.8 vol. $\%$ toluene solutions, containing 90/10 wt\% d-PMMA/P(S-alt-MMA) or 95/5 wt\% PS/P(Salt-MMA) polymer blends were solution cast onto clean glass slides and left to evaporate for one week at room temperature. The samples were then examined for turbidity to determine the miscibility of the polymer blends. Turbidity is observed when the domain size of the phaseseparated particles in a polymer blend is at least the wavelength of visible light. The interaction of the visible light with the polymer phase domains causes the light to scatter [49]. Therefore, a polymer blend that produces an opaque film is assumed immiscible and blends that produce clear films are assumed miscible.

\section{Results and discussion}

Miscibility. The miscibility of P(S-alt-MMA) in both PS and d-PMMA was examined by investigating the turbidity of solution cast films for all P(S-alt-MMA) used in this project. Each of the alternating copolymers are immiscible at $5 \%$ loading in PS as observed by the formation of opaque 
solution cast films. At 10\% loading, Alt85, Alt96, and Alt108 were miscible in d-PMMA, and at $10 \%$ loading, both Alt497 and Alt2m were observed to be immiscible in dPMMA which was indicated by the formation of opaque solution cast films. These results coincide with work by Winey on binary blends of PS or PMMA with P(S-altMMA) which showed that the P(S-alt-MMA) copolymer was more miscible in PMMA than in PS. Furthermore, Winey's work showed that the miscibility of alternating copolymers in PMMA decreased with increasing copolymer $M_{\mathrm{w}}[47]$.

Interfacial fracture toughness. Fig. 1 shows the interfacial fracture toughness of the PS/PMMA interface that is modified with the alternating copolymers studied. This figure shows the interfacial toughness, $G_{\mathrm{c}}$ of each modified interface as a function of copolymer layer thickness for the five $\mathrm{P}(\mathrm{S}$-alt-MMA) copolymers studied, where the error bars correspond to one standard deviation. The value of $G_{\mathrm{c}}$ for a bare PS/PMMA interface measured by ADCB is reported in the literature as $3.4 \mathrm{~J} / \mathrm{m}^{2}$ [34,36]. Clearly, for all 5 alternating copolymers the value of $G_{\mathrm{c}}$ increases with increased copolymer thickness at the interface.

At copolymer layer thickness of $35-50 \mathrm{~nm}$, the $G_{\mathrm{c}}$ for all $\mathrm{P}\left(\mathrm{S}\right.$-alt-MMA) are only slightly greater than the $G_{\mathrm{c}}$ for the bare PS/PMMA interface. This indicates that any improvement in $G_{\mathrm{c}}$ is merely due to the presence of any $\mathrm{P}(\mathrm{S}$-altMMA) at the interface. This result has been observed for other copolymer architectures at similar copolymer layer thickness [36].

As the copolymer layer thickness increases from 65 to $100 \mathrm{~nm}$ there is a significant increase in $G_{\mathrm{c}}$ of the interface for all alternating copolymers, with the exception of Alt85.
This region correlates to the physical condition where the copolymer forms a layer that is thick enough for the copolymer chains to penetrate into both polymers to form entanglements with each phase. The copolymer layer then serves as an anchor to which the P(S-alt-MMA) chains may interact with both of the homopolymer phases. This modification of the interface provides a mechanism to transfer stress across the phase boundary and increases the interfacial adhesion. These results agree with previous ADCB measurements on multi-block copolymers that contain block lengths that are shorter than the critical molecular weight for entanglements [34]. Results for these copolymers demonstrate that there was a continual increase in $G_{\mathrm{c}}$ with an increase in copolymer layer thickness up to $100 \mathrm{~nm}$.

As the film thickness increases to 200 and $300 \mathrm{~nm}$ there is a further, but more gradual increase in $G_{\mathrm{c}}$ of the PS/PMMA interfaces reinforced with $\mathrm{P}(\mathrm{S}$-alt-MMA) copolymers. The greatest interfacial strength for all interfaces reinforced with $\mathrm{P}(\mathrm{S}$-alt-MMA) is observed when the copolymer layer thickness is $300 \mathrm{~nm}$. This is presumably due to an increased interaction between the copolymer and both homopolymer layers as the copolymer layer thickness increases. This behavior has also been observed for ADCB measurements on PS/PMMA interfaces reinforced with $\mathrm{P}(\mathrm{S}$ ran-MMA) copolymers [22,38]. Furthermore, ADCB test by Cho on the compositionally symmetric P(S-ran-MMA) demonstrate that $G_{\mathrm{c}}$ gradually increases as the copolymer layer thickness is increased up to $900 \mathrm{~nm}$ [38]. This is surprising behavior, however, as the copolymer has formed a third layer in this regime, where there exist two separate interfaces, one between the copolymer and the polystyrene,

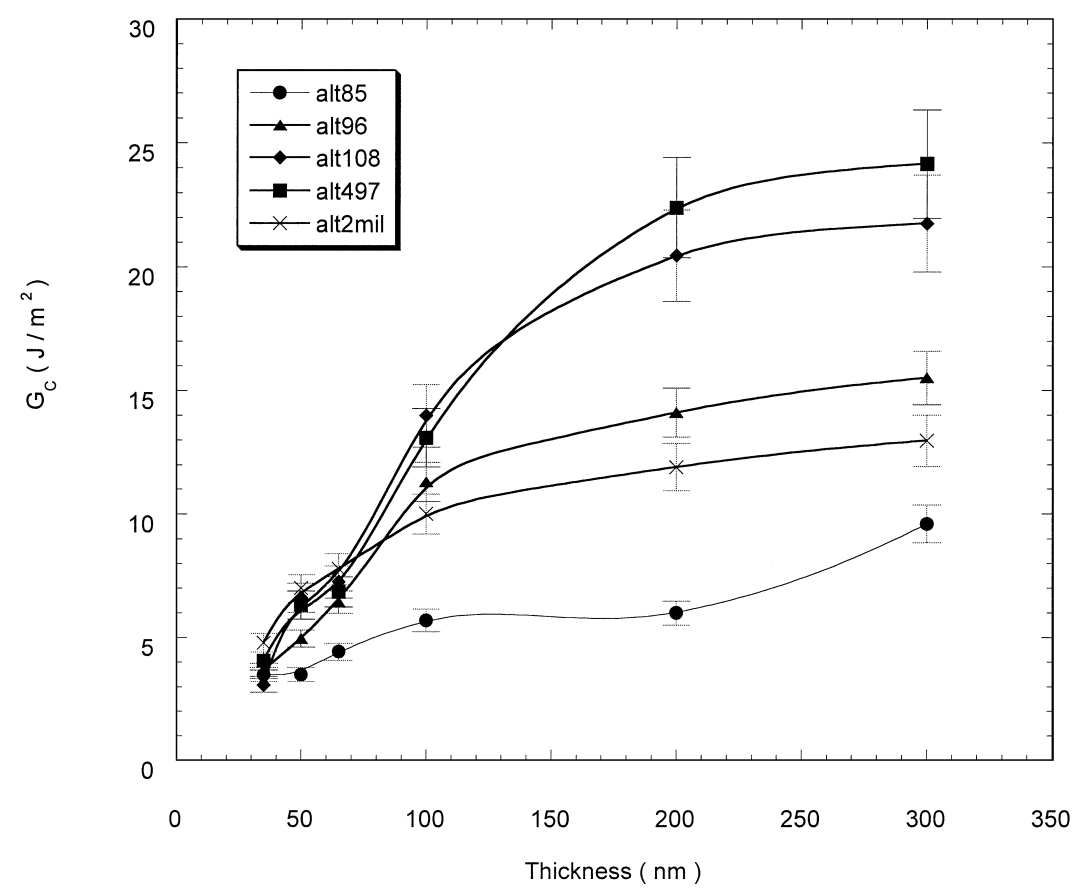

Fig. 1. The fracture toughness, $G_{\mathrm{c}}$, of the PS/PMMA interface reinforced with P(S-alt-MMA) as a function of copolymer layer thickness for five different $M_{\mathrm{w}}$. 
the other between the copolymer and poly(methyl methacrylate). To a first approximation, one would expect that the addition of more copolymer in this regime would merely separate the interfaces (i.e. create a thicker copolymer layer), but not modify their structure or their strength. Clearly this is not the case and a more thorough investigation of the structure of the interfaces for thicker copolymer layers may provide insight into this unexpected behavior.

Fig. 2 is a bar graph that shows the fracture toughness of PS/PMMA interfaces reinforced by linear styrene/methyl methacrylate copolymers $(300 \mathrm{~nm}$ thick) with varying sequence distribution [34]. The copolymers compared in this plot are very similar, all approximately 150,000 molecular weight, 50/50 copolymers, and $300 \mathrm{~nm}$ thick copolymer layers, but vary in sequence distribution. The alternating copolymer is the weakest interfacial strengthener, providing an interface with a fracture toughness of $G_{\mathrm{c}} \sim 25 \mathrm{~J} / \mathrm{m}^{2}$. The diblock, triblock, and pentablock copolymers show a $G_{\mathrm{c}}$ that increases with the number of copolymer blocks. Interestingly, these copolymers were the ones that have block lengths that are greater than the approximate entanglement molecular weight $\left(M_{\mathrm{c}}\right)$ for these polymers $(30,000)$. However, the heptablock, random and alternating produce interfaces with much lower fracture toughness, and the average 'block sizes' of these copolymers are less than the approximate entanglement molecular weight for these polymers. For these three copolymers, the $G_{\mathrm{c}}$ decreases as the sequence distribution becomes more alternating. This is interpreted as due to a decrease in the effective entanglements formed between the copolymer and the homopolymers compared to the effective entanglements formed by copolymers with block sizes greater than $M_{\mathrm{c}}$.

Copolymer molecular weight. Inspection of Fig. 1 demonstrates that the interfacial fracture toughness increases as the molecular weight of the alternating copolymer increases from 85,000 to 497,000 , but decreases

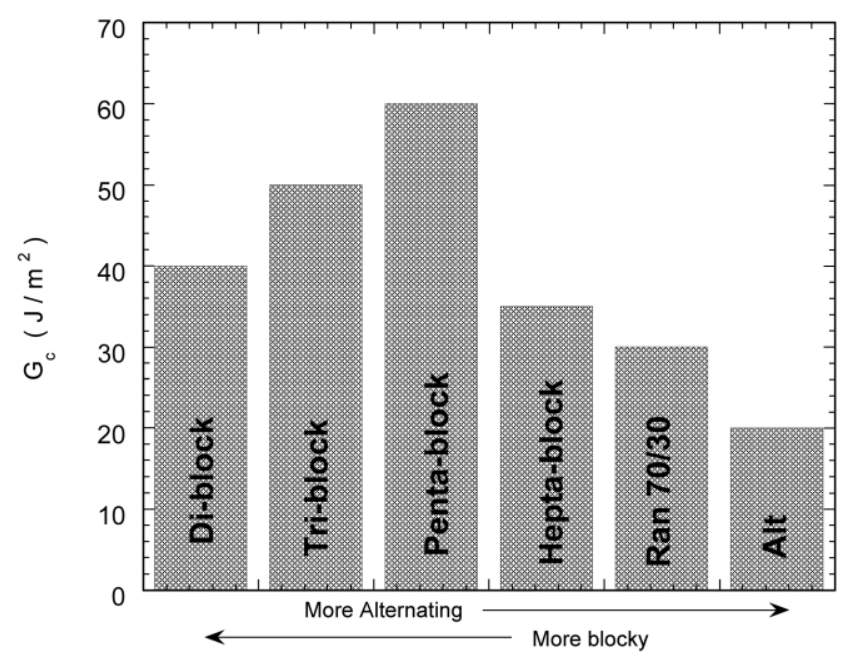

Fig. 2. A comparison of the fracture toughness for PS/PMMA interfaces reinforced with copolymers varying in sequence distribution. as the molecular weight increases to $2,000,000$. In fact, the increase in $G_{\mathrm{c}}$ with molecular weight for the 85,000, 96,000, and 108,000 Dalton copolymers appears to be almost linear, as shown in Fig. 3. This makes sense in terms of the miscibility of the copolymers in the homopolymers, as these three alternating copolymers are significantly miscible in PMMA (at least up to $10 \mathrm{wt} \%$ ), thus they are able to expand into and entangle with the homopolymers. Our interpretation of this result is that the increase in molecular weight, increases the effective number of entanglements per chain, $N_{\text {eff }}$, which has been shown to be directly responsible for the fracture toughness of a biphasic interface [13]. Also supporting this interpretation is an ADCB study by Bernard that showed that the effectiveness of $\mathrm{P}\left(\mathrm{S}_{0.7}\right.$-ran-MMA $)$ as an interfacial strengthener increases with $M_{\mathrm{w}}$ for four copolymers ranging from $1.60 \times 10^{5}$ to $4.50 \times 10^{5} \mathrm{~g} / \mathrm{mol}$ [22] as well as work by Cho which also observed an increase in $G_{\mathrm{c}}$ as a function of $M_{\mathrm{w}}$ for compositionally symmetric P(S-ranMMA) [38].

However, this explanation does not explain why the $G_{\mathrm{c}}$ for Alt497 is only slightly larger than the $G_{\mathrm{c}}$ of Alt108 nor does it explain the reduction in the $G_{\mathrm{c}}$ for Alt $2 \mathrm{~m}$ when compared to Alt497. To understand this trend, the miscibility of these larger copolymers in the homopolymers must be included in the interpretation of this anomalous behavior. Neither the Alt497 nor Alt2m are miscible in either copolymer at the concentrations studied here, suggesting that they will not expand into (nor effectively entangle with) the homopolymers to the extent of the smaller copolymers. Thus, we expect the interfaces between the Alt497 or Alt $2 \mathrm{~m}$ with either homopolymer to be relatively sharp, with little interpenetration. Moreover, if this explanation is correct, we expect that the interface of the Alt $2 \mathrm{~m}$ with the homopolymers will be sharper than those of Alt497 with the homopolymers.

To test this interpretation, NR measurements were performed on tri-layer samples that have the Alt497 and

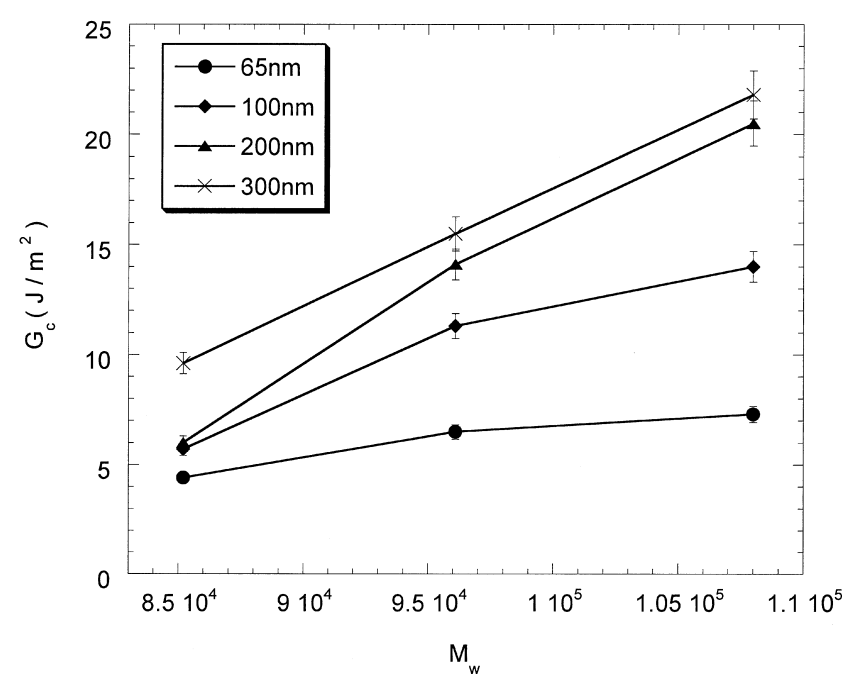

Fig. 3. A plot of the fracture toughness, $G_{\mathrm{c}}$, as a function of copolymer weight average molecular weight, $M_{\mathrm{w}}$. 
Alt2m copolymer sandwiched between d-PS and d-PMMA. Figs. 4 and 5 show the NR curves and fits for the Alt497 and Alt $2 \mathrm{~m}$ trilayer sample after two hours annealing at $150{ }^{\circ} \mathrm{C}$, respectively. In these figures, the inset corresponds to the scattering length density profile used to fit the reflectivity profile. Comparing scattering length density profiles in Figs. 4 and 5 shows that the expansion of Alt497 into the d-PS and the d-PMMA is greater for than that of Alt $2 \mathrm{~m}$.

This expansion is quantified in Table 2, which lists the properties of the copolymer layer sandwiched between the d-PS and d-PMMA, and the properties of its interfaces with the two homopolymers. In this table, the interfacial width is the thickness of the layer between the homopolymer and copolymer layers. Clearly, the Alt497 layer and its interfaces are very different from those of the Alt $2 \mathrm{~m}$ copolymer. Most notably, even though both copolymer layers were approximately $35 \mathrm{~nm}$ as cast, the Alt497 layer expands to $55 \mathrm{~nm}$, with both interfacial widths between the copolymers and the homopolymers that are greater than $25 \mathrm{~nm}$, resulting in an overall broadening of the distance between the d-PS and the d-PMMA from 35 to $110 \mathrm{~nm}$. This broadening can best be explained by the interpenetration of the copolymer with both homopolymers. This entanglement also explains the moderate $G_{\mathrm{c}}$ of the Alt497 modified interface. The scattering length density profile of the Alt $2 \mathrm{~m}$ profile also correlates well to the minimal increase in $G_{\mathrm{c}}$ by this copolymer. Whereas, the Alt497 layer broadened, the Alt $2 \mathrm{~m}$ copolymer layer thickness barely increased $(<10 \%)$ and the interfacial width between the copolymer and homopolymer remain relatively sharp $(10-15 \mathrm{~nm})$. These reflectivity results verify that the Alt $2 \mathrm{~m}$ copolymer does not significantly interpenetrate with either homopolymer, resulting in a weaker interface. This is presumably due to the limited miscibility of this large copolymer with the homopolymers.

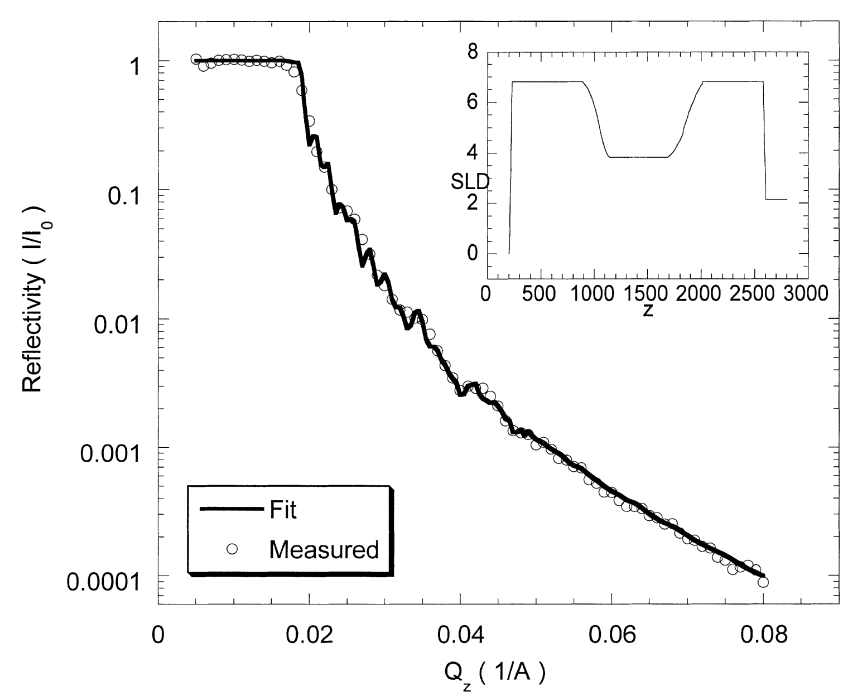

Fig. 4. Neutron reflectivity measurement and fit for the Alt497 tri-layer sample after two hours annealing. Inset corresponds to the model Scattering Length Density Profile used to fit the reflectivity profile.

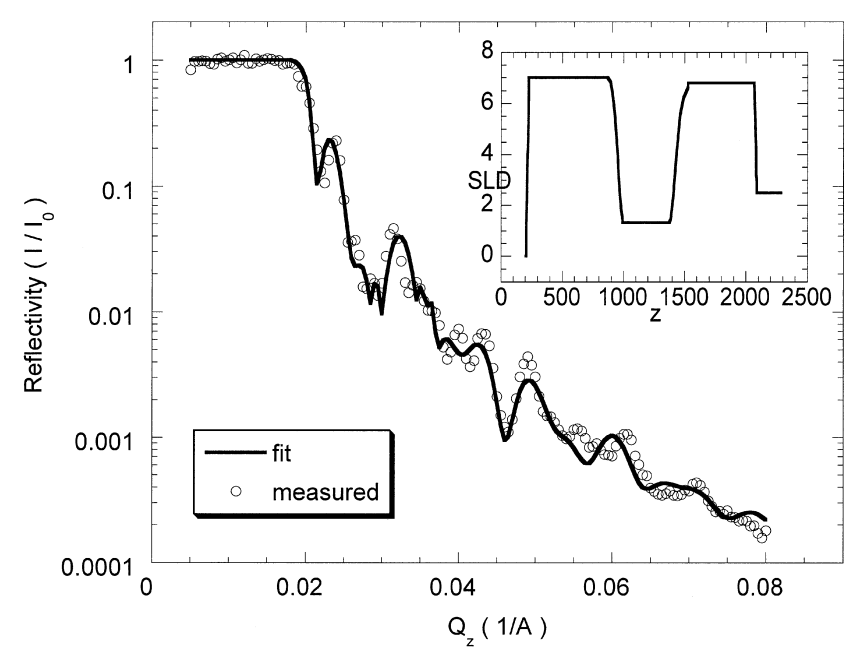

Fig. 5. Neutron reflectivity measurement and fit for the Alt $2 \mathrm{~m}$ tri-layer sample after two hours annealing. Inset corresponds to the model Scattering Length Density Profile used to fit the reflectivity profile.

The final scattering layer density (SLD) of the copolymer layer of these two copolymers also supports this interpretation. After annealing, the SLD of Alt497 increases to $3.83 \times 10^{-6} \AA^{-2}$ due to the significant presence of deuterated homopolymer in this layer, while the Annealed Alt2m layer SLD remains very low, indicating a layer that is primarily protonated.

Thus, the effect of molecular weight of an alternating copolymer on its ability to strengthen an interface is a balance of the increase in the number of entanglements per chain as the chain becomes longer and the decreased miscibility of the copolymer with the surrounding homopolymers with molecular weight. In the copolymers studied, this balance is optimized for the copolymers with $M_{\mathrm{w}}=$ 497, 000. As the sequence distribution of a copolymer will impact its miscibility, this optimization will differ for other PS/PMMA copolymers and must be determined independently.

\section{Conclusion}

Results of ADCB experiments are presented which document the ability of alternating copolymers to strengthen a polymer/polymer interface. The results demonstrate that a styrene/methyl methacrylate alternating copolymer is the least effective sequence distribution of a linear copolymer at strengthening the polystyrene/poly(methyl methacrylate)

Table 2

Density profile results of annealed interfaces

\begin{tabular}{lcc}
\hline & Alt497 & Alt2m \\
\hline Layer thickness, $L(\AA)$ & 550 & 380 \\
Scattering length density $\left(\times 10^{-6} \AA^{-2}\right)$ & 3.83 & 1.22 \\
Interfacial width $(\AA)$ PMMA/P(S-alt-MMA) & 290 & 162 \\
Interfacial width ( $)$ P(S-alt-MMA)/PS & 260 & 124 \\
\hline
\end{tabular}


interface. Interestingly, the ability of an alternating copolymer to strengthen the polystyrene/poly(methyl methacrylate) interface does not increase with molecular weight, as one might expect from a pure entanglement argument. Rather the interfacial strength also depends strongly on the miscibility of the alternating copolymer with the two homopolymers and is a balance of the change in miscibility (decrease) and entanglement per chain (increase) of the copolymer as the chain becomes longer.

\section{Acknowledgements}

The authors would like to thank 3M (Young Faculty Award) and the National Science Foundation (CAREERDMR-9702313, DMR-0241214) for financial support, which funded this research. We also acknowledge the support of the National Institute of Standards and Technology, U.S. Department of Commerce, in providing the neutron research facilities used in this work.

\section{References}

[1] Datta S, Lohse DJ, Polymeric compatibilizers: uses and benefits in polymer blends, Cincinatti, OH: Hanser; 1996.

[2] Sperling LH. Polymeric multicomponent materials: an introduction. New York, NY: Wiley; 1997.

[3] Di Lorenzo ML, Frigione MJ. J Polym Engng 1997;17:429.

[4] Dadmun MD. Macromolecules 1996;29:3868.

[5] Dadmun MD. Computational studies, nanotechnology, and solution thermodynamics of polymer systems. New York, NY: Kluwer Academic; 2000

[6] Dadmun M. Macromol Theory Simul 2001;10:795.

[7] Noolandi J. Makromol Chem Theory Simul 1992;1:295.

[8] Yeung C, Balazs A, Jasnow D. Macromolecules 1992;25:1357.

[9] Lyatskaya Y, Gersappe D, Balazs A. Macromolecules 1995;28:6278.

[10] Lyatskaya Y, Balazs A. Macromolecules 1996;29:7581.

[11] Edgecombe BD, Stein JA, Frechet JM, Xu Z, Kramer EJ. Macromolecules 1998;31:1292.

[12] Dai C, Jandt KD, Dhamodharan RI, Slack NL, Dai KH, Davidson WB, Kamer EJ, Hui C. Macromolecules 1997;30:549.

[13] Creton C, Kramer EJ, Hui C, Brown HR. Macromolecules 1992;25: 3075.

[14] Dai C, Dair BJ, Dai KH, Ober CK, Kramer EJ, Hui C-Y, Jelinski LW. Phys Rev Lett 1994;73:2472.

[15] Dai C, Chinedum O, Klaus J, Jandt B, Dair BJ, Ober CK, Kramer EJ, Hui C-Y, Jelinski LW. Macromolecules 1997;30:3542.

[16] Benkoski JJ, Fredrickson GH, Kramer EJ. J Polym Sci, Part B: Polym Phys 2001;39:2363.
[17] Xiao F, Hui C, Washiyama J, Kramer EJ. Macromolecules 1994;27: 4382.

[18] Xiao F, Hui C, Kramer EJ. J Mater Sci 1993;28:5620.

[19] Smith JW, Kramer EJ, Xiao F, Hui CY, Reichert W, Brown HR. J Mater Sci 1993;28:4232.

[20] Xiao F, Hui C, Washiyama J, Kramer EJ. Macromolecules 1994;27: 4382.

[21] Russell TP, Brown HR, Hawker CJ, Mayes AM, Kulasekere H, Kaiser H, Ankner JF. Macromolecules 1996;29:5493.

[22] Bernard B, Brown HR, Hawker CJ, Kellock AJ, Russell TP. Macromolecules 1999;32:6254.

[23] Fayt R, Jérôme R, Teyssié Ph. J Polym Sci: Polym Lett Ed 1981;19: 79.

[24] Fayt R, Jérôme R, Teyssié Ph. J Polym Sci: Polym Phys Ed 1981;19: 1269.

[25] Fayt R, Jérôme R, Teyssié Ph. J Polym Sci: Polym Phys Ed 1982;20: 2209.

[26] Fayt R, Jérôme R, Teyssié Ph. J Polym Sci: Polym Phys Ed 1989;27: 775.

[27] Fayt R, Jérôme R, Teyssié Ph. Makromol Chem 1986;187:837.

[28] Riess G, Periard J, Jolivet Y. Angew Chem-Int Ed 1972;11:339.

[29] Riess G, Jolivet Y. Adv Chem Ser 1975;142:243.

[30] Riess G, Schlienger M, Marti S. J Makromol Sci-Phys 1980;B17: 355.

[31] Gaillard P, Ossenbachstauter M, Riess G. Makromol Chemie-Rapid Commun 1980;1:771.

[32] Riess G. Makromol Chemie-Macromol Chem Phys 1985;13:157.

[33] Milner ST, Fredrickson GH. Macromolecules 1995;28:7953.

[34] Eastwood E, Dadmun M. Macromolecules 2002;35:5069.

[35] Brown HR, Char K, Deline VR, Green PF. Macromolecules 1993;26: 4155.

[36] Sikka M, Pellegrini NN, Schmitt EA, Winey KI. Macromolecules 1997;30:445.

[37] Char K, Brown HR, Deline VR. Macromolecules 1993;26:4164.

[38] Cho K, Ahn TO, Ryu HS, Seo KH. Polymer 1996;37:4849.

[39] Jones RA, Richards RW. Polymer at surfaces and interfaces. New York, NY: Cambridge University Press; 1999.

[40] Wu S. Polymer interface and adhesion. New York, NY: Marcel Dekker; 1982.

[41] Brown HR. Macromolecules 1989;22:2859.

[42] Kanninen MF. Int J Fract 1973;9:83.

[43] Gent AN, Lai SM. J Polym Sci, Polym Phys Ed 1994;32:1543.

[44] Steenbakkers-Menting HNAM, Voets PEL, Lemstra PJ. J Adhes Sci Technol 1995;9:889.

[45] Hajichristidis N, Iatrou H, Pipas S, Pitsikalis M. J Polym Sci: Part A: Polym Chem 2000;38:3211.

[46] Pellegrini NN, Sikka M, Satija SK, Winey KI. Polymer 2000;41:2701.

[47] Winey KI, Berba ML, Galvin ME. Macromolecules 1996;29:2868.

[48] Hamilton WA, Hayter JB, Smith GS. J Neutron Res 1994;2(1):1.

[49] Kämpf G. Characterization of plastics by physical methods: experimental techniques and practical application. New York: Macmillan; 1986. 